Reprod. Nutr. Dévelop., 1988, 28 (1), 169-170.

\title{
Relations entre la composition chimique des foins de luzerne et la dégradabilité in sacco de leurs matières azotées (*)
}

\author{
María R. ALVIR, J. GONZALEZ, A. ARGAMENTERIA
}

Dpto de Nutrición animal, Escuela Técnica Superior de Ingenieros Agrónomos, Universidad Politécnica, Madrid 28040, Espana.

Summary. The kinetics of nitrogen degradation in nylon bags of 10 lucerne hays were determinated in order to separate the fractions of soluble, potentially degradable and indegradable rumen-nitrogen. No relationship was found between the two former fractions and the chemical composition of the hays, but non degradable nitrogen was related to cellwall materials ; this relationship was most accurately determined with the lignin content $\left(P<0.001 ; R^{2}=0.87\right)$.

Ce travail a pour but d'étudier les variations de la cinétique de dégradation in sacco de l'azote des foins de luzerne en essayant de les mettre en relation avec celles de la composition chimique.

Matériel et méthodes. Sur 10 foins de luzerne d'origine et de composition chimique différentes, on a mesuré (tabl. 1): la solubilité de l'azote après $2 \mathrm{~h}$ d'incubation dans la salive artificielle de McDougall (1948), la teneur en constituants azotés non protéiques (Unilever, 1970) et les cinétiques de dégradation de l'azote dans le rumen de 4 moutons munis d'une canule et nourris ad libitum avec un foin de luzerne de composition moyenne.

Les foins furent fanés au sol par beau temps pendant 4 jours et conservés avec plus de $84 \%$ de matière sèche. Des sachets $115 \times 7 \mathrm{~cm}$ et pores de $12 \times 100 \mu \mathrm{m})$ contenant $3,5 \mathrm{~g}$ de foin broyé à la grille de $2 \mathrm{~mm}$, ont été placés par groupes de 4 (3 échantillons et 1 témoin) pendant 1, 3,6, 9, 15, 24 et $48 \mathrm{~h}$ dans le rumen. La quantité d'azote résiduel a été déterminée sur chaque sachet et les résultats corrigés par analyse de covariance, selon les valeurs du foin témoin.

L'évolution de l'azote résiduel a été ajustée au modèle : $Y(t)=A+B e^{-C t}$, équivalent au modèle proposé par Orskov et McDonald (1979). Dans l'équation utilisée, $A$ et $B$ représentent respectivement les fractions d'azote non dégradable et lentement dégradable par les microorganismes du rumen, $C$ correspond au taux horaire de dégradation et la différence $100-(A+B)$ représente la fraction d'azote alimentaire rapidement disparue par solubilisation. Pour plusieurs foins, l'existence d'un temps de latence $\left(t_{0}\right)$ a nécessité la transformation du modèle initial en : $Y(t)=A+B$; pour $t<t_{0}$ et $Y(t)=A+B^{\prime} e^{-C t}$ pour $t>t_{0}$, la valeur $A+B$ correspondant à l'azote résiduel après $1 \mathrm{~h}$ d'incubation. 
Résultats et discussion. La répartition de l'azote des foins en azote soluble, potentiellement dégradable et azote non dégradable est rapportée dans le tableau 1. Pour 6 des 10 foins on a constaté l'existence d'un temps de latence de 3 à $6 \mathrm{~h}$. La proportion d'azote non dégradable (A) présente une corrélation positive avec les teneurs en parois (NDF), lignocellulose (ADF) et lignine (ADL) mais non avec l'azote du NDF. Les relations sont d'autant plus étroites que la fraction des constituants pariétaux étudiée est moins digestible, comme le montrent les équations suivantes :

$A=-31,86+2,04 \times \mathrm{NDF}-0,02 \times \mathrm{NDF}^{2} ; \mathrm{R}^{2}=0,50 ; \mathrm{P}<0,05$
$\mathrm{~A}=-9,92+0,44 \times \mathrm{ADF}+0,01 \times \mathrm{ADF}^{2} ; \mathrm{R}^{2}=0,78 ; \mathrm{P}<0,001$
$\mathrm{~A}=\quad 6,37-3,70 \times \mathrm{ADL}+0,74 \times \mathrm{ADL}^{2} ; \mathrm{R}^{2}=0,87 ; \mathrm{P}<0,001$

La fraction d'azote rapidement disparu n'est liée ni à la teneur en constituants azotés non protéiques, ni à la solubilité de l'azote dans la salive artificielle. II n'y a pas nons plus de relation entre la proportion d'azote dégradable (B) et la teneur en azote des foins.

Les taux de dégradation (C), qui ont été très différents entre les foins, sont en relation négative avec la proportion $\mathrm{d}^{\prime}$ azote lentement dégradable ( $\mathrm{B}$ ) : $\mathrm{C}(\% / \mathrm{h})=29,2-0,427 \times \mathrm{B}(\%) ; \mathrm{R}^{2}=0,70 ; \mathrm{P}<0,01$.

Cette relation est difficile à interpréter. Elle pourrait résulter de différences dans le rapport entre le nombre de bactéries attachées sur les particules du foin et la teneur de celles-ci en azote dégradable. En plus, le temps de latence doit influencer l'attachement de bactéries et par conséquent les taux de dégradation.

TABL. 1. - Composition chimique et cinétique de dégradation de l'azote.

\begin{tabular}{lcccccccccc}
\hline Foins & 1 & 2 & 3 & 4 & 5 & 6 & 7 & 8 & 9 & 10 \\
\hline Numéro et stade de la coupe & $1-\mathrm{V}$ & $2-\mathrm{V}$ & $2-\mathrm{B}$ & $2-\mathrm{F}$ & $4-\mathrm{F}$ & $1-\mathrm{DF}$ & $1-\mathrm{F}$ & $1-\mathrm{DF}$ & $3-\mathrm{DF}$ & $2-\mathrm{F}$
\end{tabular}

Matières azotées totales

(MAT)

Parois (NDF)

Lignocellulose (ADF)

Lignine (ADL)

$\begin{array}{rrrr}28,2 & 24,4 & 20,2 & 19,8 \\ 21,9 & 28,1 & 36,6 & 38,3 \\ 19,7 & 23,2 & 27,4 & 33,0 \\ 3,5 & 4,8 & 5,3 & 6,5\end{array}$

$\%$ de la matière sèche

\begin{tabular}{|c|c|c|c|c|c|c|c|c|c|c|}
\hline \multirow{4}{*}{$\begin{array}{l}\text { Azote soluble } \\
\text { Azote non protéique (ANP) } \\
\text { Azote du NDF (N. NDF) }\end{array}$} & \multicolumn{10}{|c|}{$\%$ de l'azote total } \\
\hline & 42,5 & 42,9 & 30,0 & 30,1 & 32,7 & 32,3 & 29,9 & 37,9 & 43,4 & 30,5 \\
\hline & 28,5 & 35,3 & 25,0 & 23,2 & 27,5 & 23,0 & 18,4 & 30,9 & 33,8 & 22,9 \\
\hline & 6,2 & 8,8 & 22,8 & 18,8 & 14,5 & 29,8 & 13,0 & 11,5 & 13,5 & 18,0 \\
\hline \multirow{6}{*}{$\begin{array}{l}\text { Temps de latence }(\mathrm{h}) \\
\text { Azote rapidement disparu (\%) } \\
\text { Azote lentement dégradable } \\
(\mathrm{B} ; \%) \\
\text { Azote indégradable }(\mathrm{A} ; \%) \\
\text { Taux de dégradation } \\
(\mathrm{C} ; \% / \mathrm{h})\end{array}$} & - & - & 6 & 3 & - & 3 & 3 & - & 3 & 3 \\
\hline & 44,3 & 41,8 & 40,9 & 43,3 & 36,1 & 35,8 & 48,1 & 39,2 & 39,0 & 38,4 \\
\hline & 5 & & & & & & & & & 6 \\
\hline & 29 & 3,9 & $\begin{array}{r}51,0 \\
7,8\end{array}$ & $\begin{array}{l}41,4 \\
15,4\end{array}$ & $\begin{array}{l}31,4 \\
12,5\end{array}$ & $\begin{array}{r}3,2 \\
8,9\end{array}$ & $\begin{array}{l}30,1 \\
132\end{array}$ & $\begin{array}{l}0,0 \\
10,9\end{array}$ & 147 & 200 \\
\hline & & & & & & & & & & \\
\hline & 6,6 & 6,5 & 6,4 & 9,2 & 7,7 & 6,0 & 15,1 & 6,5 & 12,1 & 9,7 \\
\hline
\end{tabular}

${ }^{*} \mathrm{~V}$ : Végétatif ; $\mathrm{B}$ : Bourgeonnement ; DF : Début floraison ; F : Floraison.

McDougall E. I., 1948. Biochem. J., 43, 99-106.

Ørskov E. R., McDonald I., 1979. J. agric. Sci. Camb., 92, 499-503.

Unilever Research Laboratory, 1970. Colworth/Welwyn. Method $n^{\circ} \mathrm{R} / \mathrm{C} / 2^{\circ}$. 